\section{EVALUATION OF TWO TYPES OF DRUG TREATMENT WITH QEEG IN CHILDREN WITH ADHD}

Aims: The aim of this study is to evalute the effects of methylphenidate and atomoxetine treatments on electroencephalography (EEG) signals in volunteer children diagnosed with Attention Deficit and Hyperactivity Disorder(ADHD).

Methods: The study contained 40 children all of whom were between the ages of 7 and 17 . The participants were classified into two groups as ADHD $(n=20)$, which was in itself divided into two groups as ADHD-MPH (ADHD- Metylphenidate treatment) $(n=10)$ and as ADHD-ATX (ADHD-Atomoxetin treatment) ( $n=10)$, and one control group ( $n=20)$. Following the first EEG recordings of the ADHD group, long-acting methylphenidate dose was applied to one ADHD group and atomoxetine dose was applied to the other ADHD group. The effect of optimal dosage is about for 4-6 weeks in general. Therefore, the response or lack of response to the treatment was evaluated three months after the beginning of the treatment.

After methylphenidate and atomoxetine drug treatment, in order to obtain mean and maximum power values for delta, theta, alpha and beta band, the EEG data were analyzed.

Results: The EEG power spectrum densities in all the bands yielded similar findings in both methylphenidate and atomoxetine. Although statistically significant frequency values of the electrodes were amplitude and maximally varied, in general, they appeared mostly at both frontal and temporal regions for methylphenidate and atomoxetine.

Conclusion: Especially, after atomoxetine treatment, Quantitative Electroencephalography (QEEG) rates at frontal area electrodes were found statistically more significant than methylphenidate QEEG rates. What has been researched in this study is not only whether QEEG is likely to support the diagnosis, but whether changes on QEEG by treatment may be related to the severity of ADHD as well.

Keywords

- Attention-deficit hyperactivity disorder $(\mathrm{ADHD}) \cdot$ stimulants $•$ non-stimulants $•$ drug treatment $•$ QEEG
Ramazan Aldemir',

Esra Demirci²,

Ayşe Kaçar Bayram ${ }^{3}$

Mehmet Canpolat ${ }^{3}$

Sevgi Ozmen²,

Hüseyin Per ${ }^{3}$,

Mahmut Tokmakci ${ }^{4}$ *

Biomedical Device Technology Program, Erciyes University

${ }^{2}$ Department of Child Psychiatry, School of

Medicine, Erciyes University

${ }^{3}$ Department of Pediatrics, Division of Pediatric Neurology, School of Medicine, Erciyes University

${ }^{4}$ Biomedical Engineering Department, Erciyes University

Received 03 May 2018 accepted 21 June 2018

\section{Introduction}

It is known that attention deficit and hyperactivity disorder (ADHD), if not treated, not only affect the functionality in childhood period of patients, but it can cause social and educational problems in later periods of their lives, as well. Therefore, it is very important for the disease to be diagnosed and treated at early ages. Although stimulants have been widely used for the treatment of ADHD, the drugs that have been used for this purpose clinically about $20 \%$ of children do not respond to the treatment. Barry et. al., taking EEG analysis into consideration, investigated the effect of acute dose of atomoxetine, one of the stimulants, on the performance of children with ADHD. They analyzed absolute and relative power values for all brain bands and compared the children with ADHD and a control group. They revealed that atomoxetine provided significant increases in absolute and relative beta, and caused several topographical changes in other bands[1]. Chiarenza et al. showed that there was an increased activation in some cerebral lobes that responded to the treatment of atomoxetine. Despite the continued increase in children who did not respond to treatment with atomoxetine, this increased activation, 6 and 12 months after the use of atomoxetine in treatment response, led to a decrease in children. They reported similar findings obtained through different techniques supporting the evidence regarding that these cerebral areas are involved in the pathophysiology of ADHD[2].

In a study performed by using atomoxetine, it was reported that when ADHD patients administered this drug were compared with a sub-group with ADHD without the drug, it was found that their EEG activity was abnormal. In addition, it was also reported that the children who had ADHD at the beginning of their study showed QEEG abnormalities which have taken part in literature, and that through their study, new data were obtained to support the chronic effects of atomoxetine on QEEG of children with ADHD. Moreover, treatment with atomoxetine has been shown to be effective in different subgroups of ADHD[3]. In another study in which methylphenidate was used, 
they found some significant effects on ADHD subgroups[4]. It was reported in one other study that there was a deceleration in alpha waves after the application of a single dose of methylphenidate to ADHD subgroups. These findings emphasized that the main frequency of the alpha band may be a determinant index to satisfy methylphenidate response for ADHD[5].

There are studies showing that EEG profiles are significantly normalized by EEG changes and acute atomoxetine treatment resembling the changes caused by psychostimulants (methylphenidate /dexamphetamine) [6-9]. It was reported in some studies that QEEG profiles of those who responded or not responded to the treatment are declared to have defined the QEEG sub-groups of the children with ADHD[1, 7, 10-21]. In a study on the investigation of the effects of medical treatment process of the children with cognitive and behavioral disorders accompanying ADHD on EEG, an increase alpha activity in central and parietal regions in cognitive activation conditions is observed. It was shown that there was an increase in the beta activity at the frontal area in those who responded to the treatment and a decrease in the beta activity at the same region in those who did not respond to the treatment. In addition, in children with ADHD, stimulant medication is determined to have increased beta activity, especially in the frontal areas[22]. DongHoSong et al. determined in their study that methylphenidate caused a significant increase in the alpha band in the right and left frontal and occipital areas; an increase in the beta band in almost all areas except the temporal region; a decrease in the theta band in the occipital and right temporo-parietal regions; a slight decrease in gamma activities in occipital-parietal regions, and an increase in the rate of right frontal and parieto-occipital regions (i.e. theta / beta ratio)[23].

The aim of this study is to investigate the therapeutic properties of long-acting methylphenidate and atomoxetine drugs commonly used in ADHD with EEG signals in conjunction with classical medical evaluation. It also aims to reveal the advantages and disadvantages of these two drugs relative to each other. Moreover, whether or not the treatment response and the relationship of resistance with EEG signal differences is to be determined by clinical follow-up and scale evaluation can help to guide the clinician in the selection of treatment for EEG signal parameters according to the obtained results.

\section{Subjects and Methods}

Fourty volunteer children, directed from the Faculty of Medicine Child Psychiatry to the Department of Child Neurology at Erciyes University in Turkey, newly diagnosed with ADHD according to Diagnostic and Statistical Manual of Mental Disorders-IV (DSM-IV) took part in our study. The participants were classified into two groups as $A D H D(n=20)$, which was in itself divided into two groups as $A D H D-M P H$ (ADHD- Metylphenidate treatment) $(n=10)$ and as ADHD-ATX (ADHD-Atomoxetin treatment) $(n=10)$, and one control group $(n=20)$. Twenty 7-17-year-old right-handed participants were identified by using the Kiddie-Schedule for Affective Disorders and Schizophrenia for School Age Children (K-SADS) and Wechsler Intelligence Scale for Children-Revised (WISC-R) tests to assess intelligence and psychiatric exclusion criteria. Those patients who had other medical conditions and who used medicine were not included in the study. The control group was one to one matched with the patient group in terms of age and sexuality, and twenty healthy volunteers, evaluated with WISC-R, without mental retardation, were included in the study. The demographic characteristics/ profiles of the participants are given mean age, years of education, and WISC-R mean scores for ADHD that uses MPH and for ADHD that uses ATX, respectively as follows: The mean age (Standart Deviation: \pm SD) of the ADHD (MPH), the ADHD (ATX), and Control children's groups were $9.5( \pm 3.50)$ years, $8.3( \pm 4.20)$, and 9.1 $( \pm 2.15)$ years, respectively. The education years $( \pm$ SD) of the ADHD (MPH), the ADHD (ATX), and Control children's groups were $4.0( \pm 3.30)$ years, $3.5( \pm 4.70)$, and $3.0( \pm 1.58)$ years, respectively. The WISC-R mean scores ( \pm SD) of the ADHD (MPH), the ADHD (ATX), and Control children's groups were $93.9( \pm 15.20)$ years, $96.2( \pm 11.70)$, and $110.0( \pm 5.40)$ years, respectively. Our work was approved by the Erciyes University Clinical
Research Ethics Committee and is suitable for the Helsinki Declaration. During the study, informed consent forms were obtained from all the volunteers.

Following the first EEG recordings of the ADHD group, long-acting methylphenidate dose (OROS MPH) or atomoxetine dose was applied as $0.5 \mathrm{mg} / \mathrm{kg} /$ at the beginning of the day. And it was titrated to $1.2 \mathrm{mg} / \mathrm{kg} /$ day. The time to achieve optimal dose is about 4-6 weeks in general. Therefore, the response or lack of response to treatment were evaluated three months after the beginning of the treatment. The patients, following the treatment efficiency with the decrease of the improvement score to 2 or 1 according to CGI-I (Clinical Global Impressions), were assessed for the second time with EEG evaluation and also with the evaluation of Conners Rating Scale Parent and Teacher Form as well as with clinical interviews.

The face-to-face interviews were conducted with patients and their families every two weeks during the study to ensure the continuity of the treatment, in the cases in which this was not possible, clinical developments were followed up through telephone. Two EEGs in total, one is the pretreatment and the other is in the third month after treatment, were shot for the ADHD patients, and one EEG was shot for the control group after the first evaluation. The analysis and evaluation of the EEG signals were performed with 19 channel Comet-PLUS XL Lab-based EEG system of GRASS technology company, which is sampling for about $25-30$ minutes at $200 \mathrm{~Hz}$ for 7-17 age group patients from Neurology Department of Erciyes University. The EEG electrodes (Frontal, Temporal, Central, Parietal and Occipital) were grouped in the computer program to analyze the received data.

The data from EEG channels, after the grouping, were purified from noise and disturbing effects through butterworth filter which bandpasses in the range of $0.5 \mathrm{~Hz}$ to 64 $\mathrm{Hz}$ in the MATLAB environment. Later on, the signals purified from disturbing effects were analyzed on the frequency axis using statistical properties. The results of the study were evaluated with Matlab software (Version9.0.1, Mathworks, USA), a computer software, and Sample t-test was used in the evaluation of numerical data. The significance level was 
regarded as $p<0.05$. The stages of EEG signal processing and statistical analysing are shown in Figure 1.

In the literature survey, four artifacts correction methods were mentioned. One of these is the wavelet analysis method. The wavelet analysis method performs as a filter that can remove artifacts located in frequency bands that overlap with those of the neurological phenomena of interest. It is more advantageous than other methods because of its filter feature based on wavelet transform. Therefore, in this study the wavelet analysis method is chosen to remove artifacts from EEG signals [24-26].

After we acquired the raw EEG signal data, all EEG signals are pre-processed using Wavelet analysis. Pre-processing procedures are resampling and windowing of the EEG signal. During the measurement of EEG signals, other kinds of signals, such as ECG, EMG, $50 \mathrm{~Hz}$ electrical interference, so called artifacts are also captured by electrodes. Artifacts are undesired signals that can introduce significant changes in neurological signals and ultimately affect the neurological phenomenon [27]. Some types of artifacts increase or decrease EEG band powers (delta, theta, alpha, beta) which leads to mistakes measured in the EEG bands.

\section{Results}

The EEG signals from individuals participating in the study were grouped according to frontal, temporal, central, parietal, occipital regions, and the average power for each electrode used to receive brain signals were separately obtained for maximum power and frequency values (delta, theta, alpha and beta bands) at which maximum power were obtained.

The obtained data were subjected to t-test with the control group for statistical analysis in the Matlab program to reveal effective electrodes in four groups including before methylphenidate and atomoxetine use, and after methylphenidate and atomoxetine use, respectively. Figure 2 indicates that the mean power values of the electrodes at the frontal region, delta, theta, alpha, and beta bands were higher than those of other regional electrodes, especially to other regions of the brain, in which the EEG signals were recorded from the individuals with ADHD before methylphenidate use, and mean power values varied more especially at the delta band than other bands; showed less change at theta band compared to delta band; mean power value continued to decrease at the alpha band, and this difference was the lowest at the beta band as compared to control group. The results of the analysis of EEG records taken after using methylphenidate showed that the mean power values of the delta band, obtained were especially higher in the frontal and temporal regions of the brain, decreased and approximated to the control groups. According to the information obtained from EEG records taken before and after methylphenidate use; an exact opposite situation first started to occur in the theta band in central, parietal and occipital regions, and continued at the alpha band, and the difference increased and reached to the highest level at the beta band. Considering maximum and mean power values of the bands obtained from the EEG records, it was seen that this difference was considerably high in the delta band in the frontal region compared to the control group before methylphenidate use, and the maximum and mean power difference in reference to the control group was zeroized after methylphenidate use, this effect was not much in other bands, and the increase in total mean power values especially in the beta band in the parietal and occipital regions after methylphenidate use was shown in Figure 2. According to the results of this analysis, it can be said that methylphenidate has a therapeutic effect.

According to the analysis results of EEG records taken before and after atomoxetine use; the mean power values of delta band obtained decreased, especially in the frontal and temporal regions of brain approximate to the control group. This situation showed similarity to the effect obtained before and after Methylphenidate use. But, more

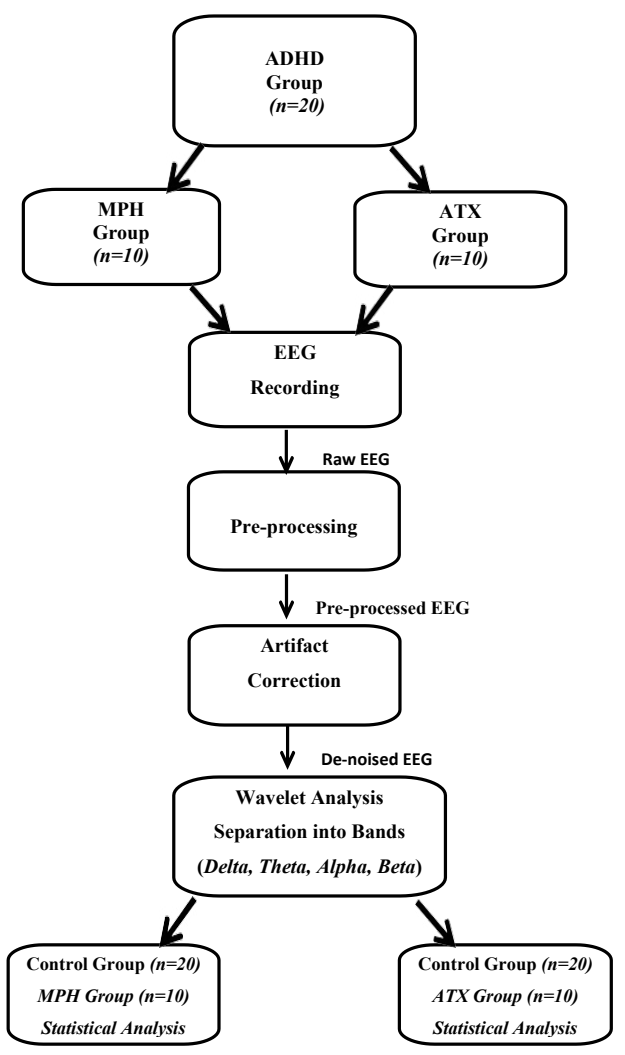

Figure 1. EEG signal processing and statistical analysis stages. 


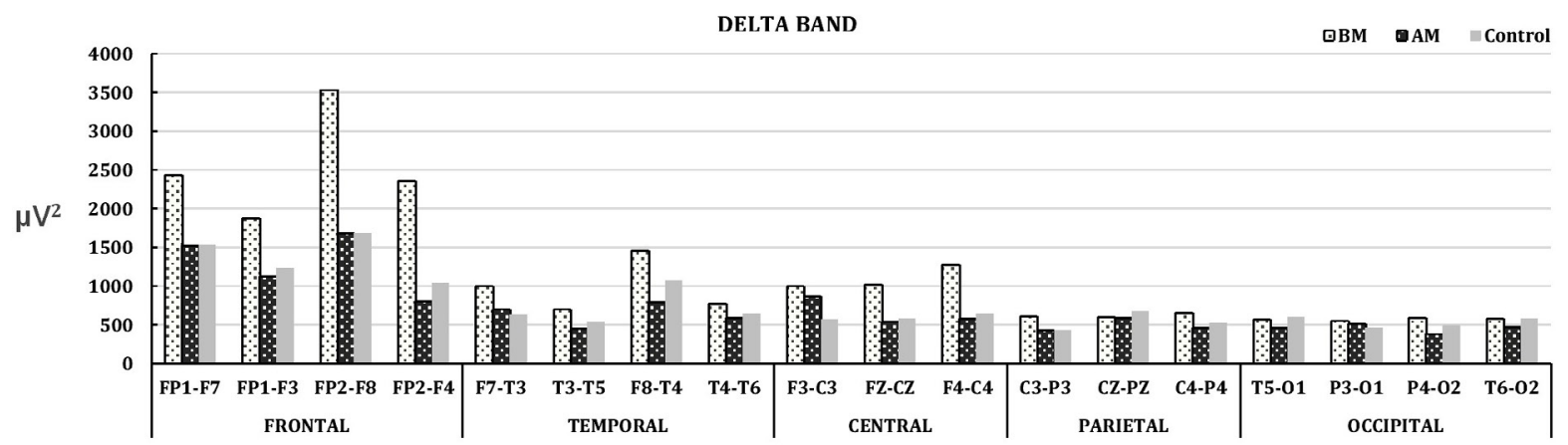

(a)

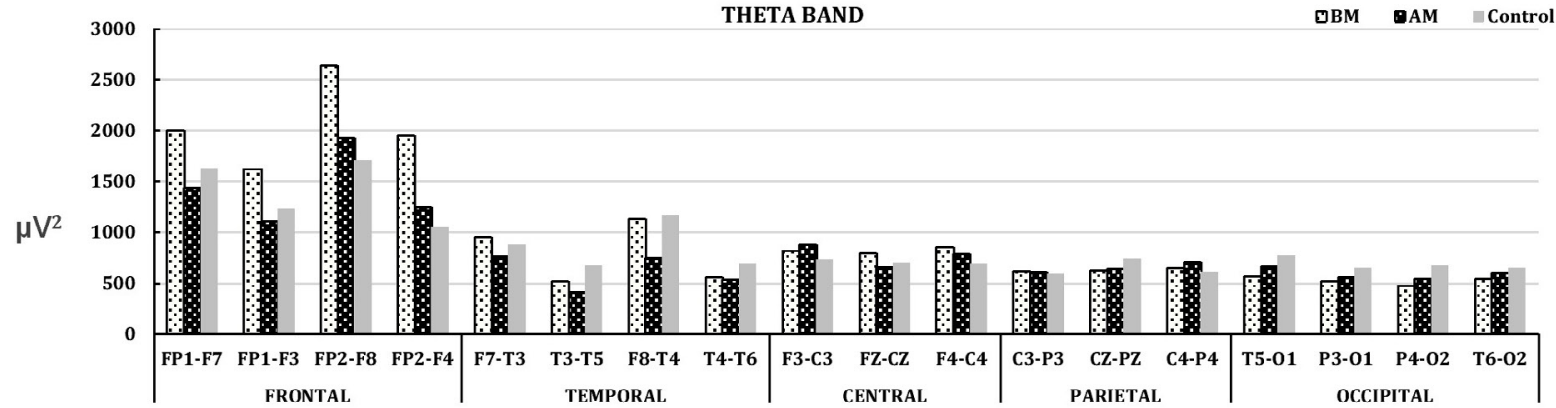

(b)

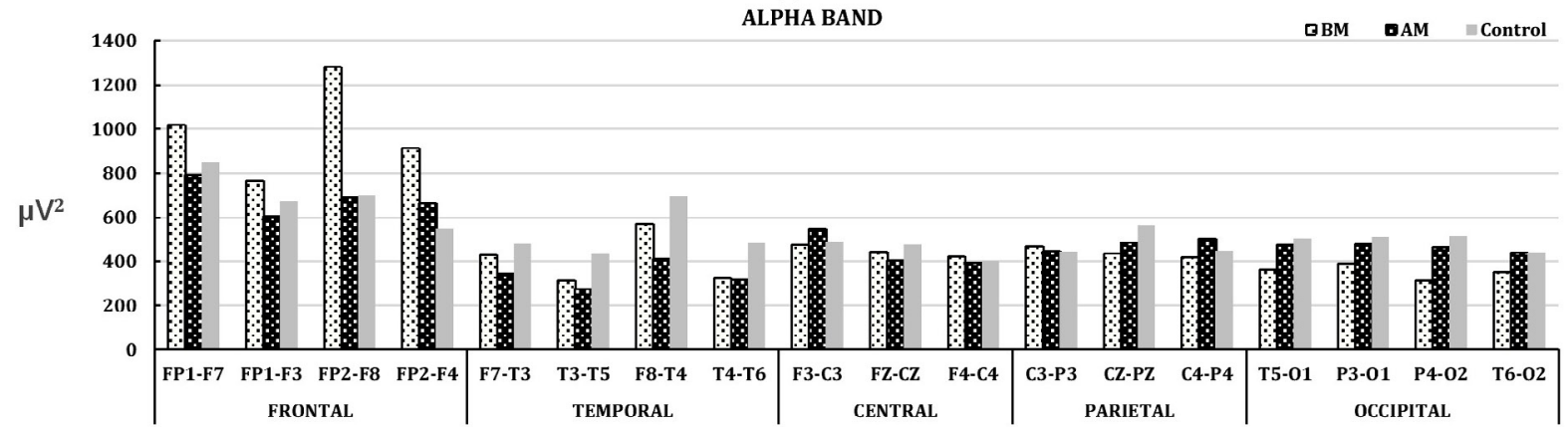

(c)

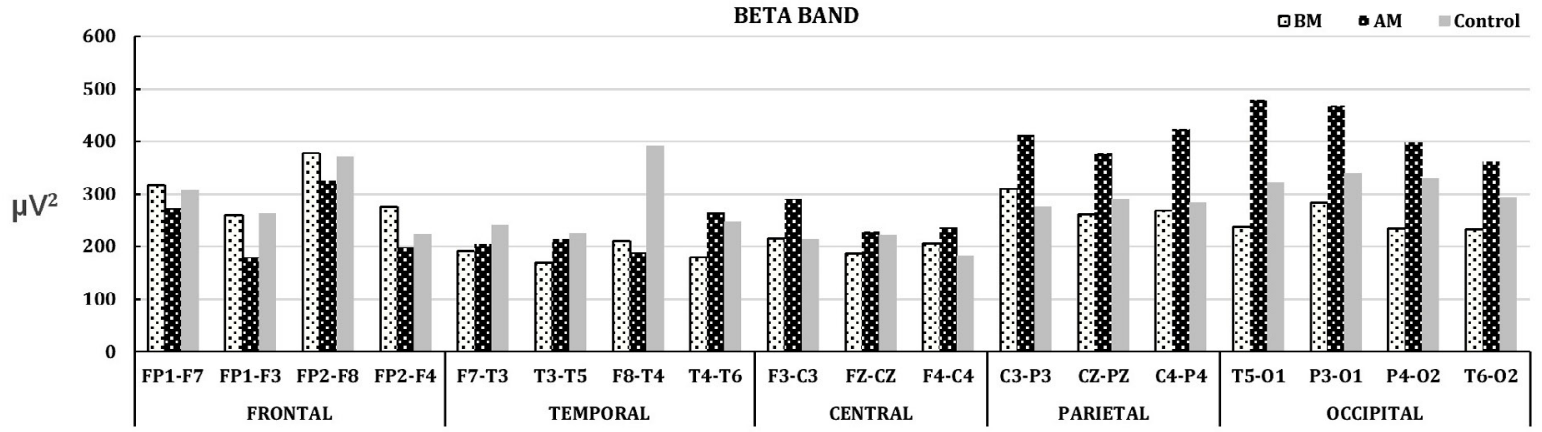

(d)

(BM: Before Methylphenidate AM: After Methylphenidate)

Figure 2. Mean power change in EEG bands between Methylphenidate using ADHD and Control groups. (a)Delta band, (b)Theta band, (c)Alpha band, and (d)Beta band 
change value was obtained at the mean power value in the frontal region of the delta band after atomoxetine use compared to after methylphenidate use. According to the information obtained from EEG records before and after methylphenidate use; an exact opposite situation first started at theta band in central, parietal, and occipital regions, and continued along alpha band, and difference increased at beta band reaching to the highest point. Considering total average power values of the bands obtained from the EEG records; it was seen that this difference at the delta band in the frontal region was significantly greater than the control group before atomoxetine use, and the total maximum power difference in reference to the control group was zeroized after atomoxetine use, and this effect was not seen much in other bands, and an increase in the mean power was observed especially at the beta band in the parietal and occipital regions. According to these results, it can be said that the treatment of atomoxetine has a therapeutic effect. This result obtained shows similarity to the one obtained after methylphenidate use. The change showing adverse effect at the mean power in parietal and occipital regions of the brain after the atomoxetine use was less than the change at the mean power obtained after the methylphenidate use. These changes obtained are shown in Figure 3.

Considering statistical significance $(p<0.05)$ values obtained because of the analysis before and after methylphenidate use, it was seen that the frequency value at which FP1-F3 electrode had maximum power at delta, alpha and beta bands obtained. Similarly, the frequency values at which F7-T3 electrode had maximum power at alpha band, FP2-F4 electrode at delta band, T4-T6 electrode at theta band, F3-C3 electrode at delta band, F8-T4 electrode at beta band, C4-P4 electrode at delta band, P4-O2 electrode at theta band, $\mathrm{T} 6-\mathrm{O} 2$ electrode at beta band were found significant. But these frequency values at the bands vary. T4-T6 electrode was found significant at the maximum frequency value at theta band, and at the mean power and maximum power value at beta band. $\mathrm{Cz}$ $\mathrm{Pz}$ electrode was found significant at mean power and maximum power values at beta band. C4-P4 electrode was found significant at maximum power value at delta and beta bands, and at mean power value at beta band. P4-O2 electrode was found significant at maximum power value at theta and alpha bands, and at mean power and maximum power values at beta band. Besides, T5-01 electrode was found significant at mean power value at beta band, and the association of these values with EEG electrodes were indicated in Figure 4(a), and their statistical significance values were indicated in top of the Table 1.

Considering statistical $(p<0.05)$ significance values because of the analysis before and after atomoxetine use, it is seen that the frequency value at which FP1-F7 electrode had maximum amplitude at delta and theta bands. Similarly, significant values were obtained at the frequency values at which maximum amplitude was obtained at CZ-PZ and C4-P4 delta bands. Statistical significant values were obtained at the mean power values at T3-T5, F3-C3, Fz-Cz electrodes before and after atomoxetine use. Besides, the association of significant values at which only maximum amplitude was obtained with EEG electrodes in $\mathrm{P} 4-\mathrm{O} 2$ and $\mathrm{T} 6-\mathrm{O} 2 \mathrm{EEG}$ channels is given in Figure 4(b), and statistical significance values are given in middle of the Table 1.

According to the QEEG findings obtained from EEG analyzes after the use of methylphenidate; FP2-F4, FZ-CZ, C3-P3, T5-O1, $\mathrm{P} 4-\mathrm{O} 2$ channels approximated to the control group decreasing at delta/beta rate; FP2-F4, C4-P4, P4-O2 channels at both delta/alpha, and theta/delta rates are shown in Figure 5(a), and the statistical significance values obtained from these channels are shown on the left bottom of the Table 1.

According to the findings of QEEG obtained from the results of EEG analyzes after the use of atomoxetine; FP1-F7, T3-T5 and FZ-CZ channels were found statistically significant at delta / beta rates. According to the QEEG rates; T3T5, Fz-Cz, F4-C4, C4-P4 channels were found statistically significant at delta / alpha rate; FP1-F7, FP2-F4, F4-C4 channels at theta/alpha rate; FP1-F7, FP2-F4, FP1-F3, T6-O2 channels at theta/beta rate. $\mathrm{Fz}-\mathrm{Cz}$ channel was found statistically significant at theta / delta rate. QEEG rates approximated to the control group decreasing, which is shown in Figure 5(b), and statistical significance values obtained from these channels are shown on the right bottom of the Table 1.

\section{Discussion}

This study investigated the changes in EEG, caused by drugs used as stimulants in children with ADHD. The results of the study showed that ADHD group had more delta, theta and alpha activities, especially in the frontal regions. It has been shown that the stimulants used usually cause an increase in post-stimulant beta activity in the parietal and occipital regions rather than normalization of the EEG. Since children with ADHD using methylphenidate and atomoxetine were evaluated from EEG signals, the results of the analysis were discussed comparatively after methylphenidate and atomoxetine use.

i) When EEG bands were examined after methylphenidate use, it is seen that EEG power spectra decreased especially in the frontal region and approximated to the control group values. But, EEG power spectra significantly increased especially in the occipital-temporal regions about the pre-methylphenidate control group. Although some studies in the literature have shown that stimulants do not have much effect on EEG frequency band changes[28-30], in our study, the changes in EEG frequency after methylphenidate use support the similar results of many studies in the literature $[1,7,10$ 21, 31, 32].

A study investigating the effects of stimulants showed that the EEG of $56.9 \%$ of a group of children with ADHD returned to normal after stimulants were applied to them, and that the EEG of of $33.8 \%$ didn't change and that the EEG of $9.3 \%$ showed an increase in EEG abnormality [11].

In a study in which the effects of methylphenidate on EEG were investigated, it was reported that theta and alpha levels decreased, but beta activity increased in the frontal region in those who responded well to methylphenidate application, but reversed changes were observed in the EEG of those who responded poorly $[33,34]$.

ii) When the results of EEG analysis after atomoxetine use were evaluated, it was seen that EEG power spectrum densities in 

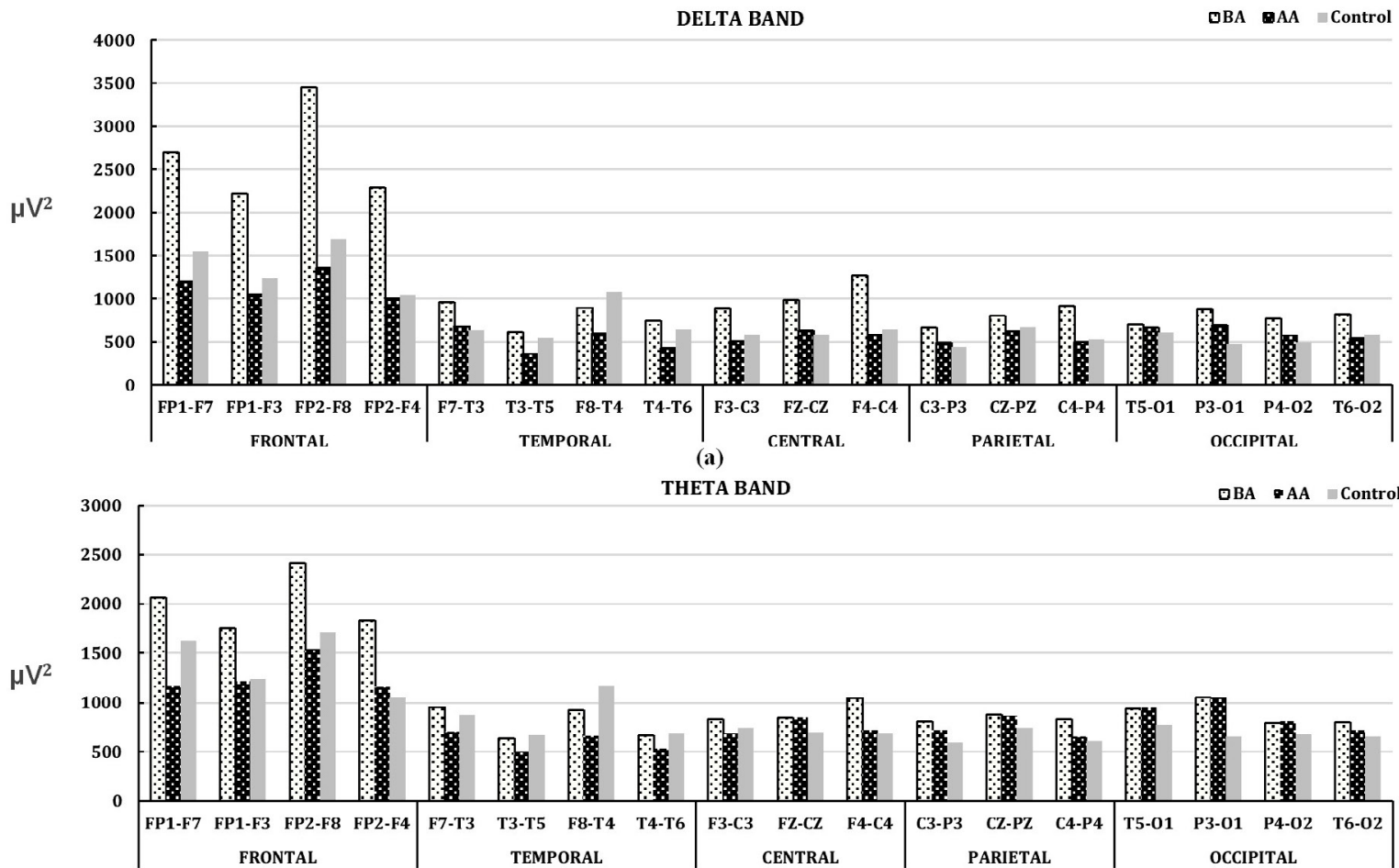

(b)

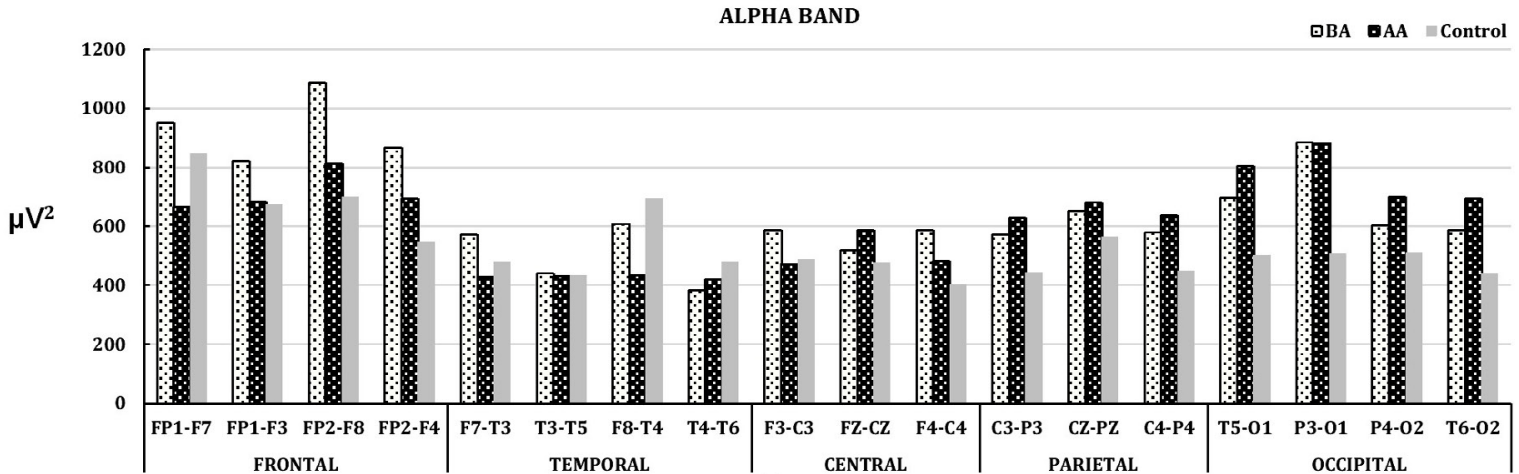

(c)

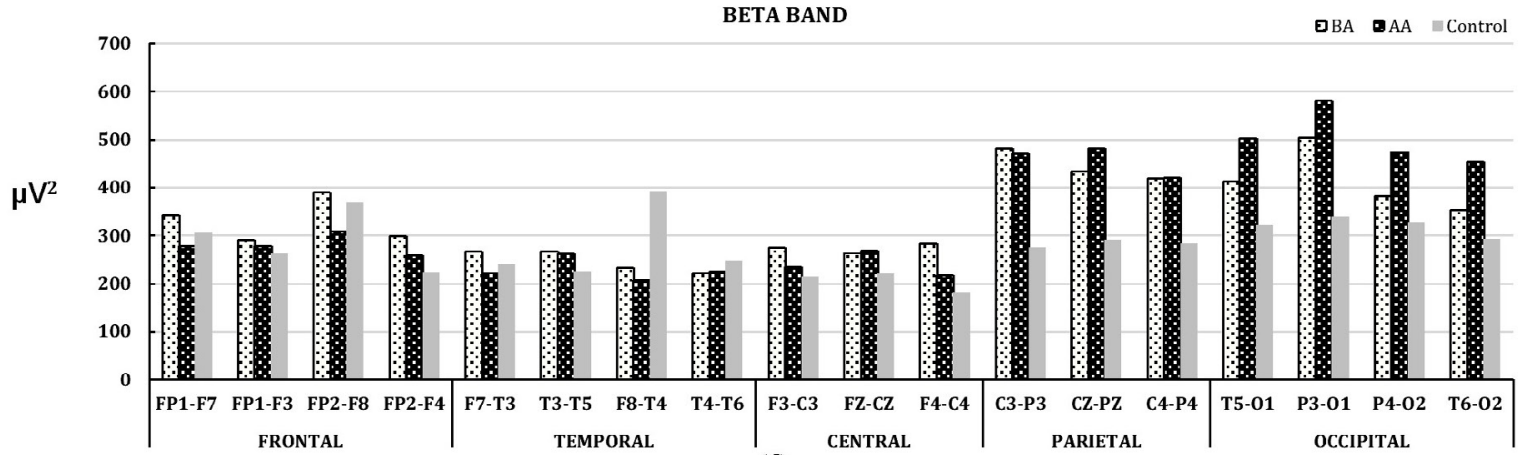

(d)

(BA:BeforeAtomoxsetineAA: After Atomoxsetine)

Figure 3. Mean power change in EEG bands between Atomoxetine using ADHD and Control groups. (a)Delta band, (b)Theta band, (c)Alpha band, and (d)Beta band 
delta, tetra, alpha and beta bands showed similarities to the findings obtained after methylphenidate use. Although the maximum power and frequencies at which electrodes were statistically significantly varied, they mostly occurred in the frontal and temporal regions for both methylphenidate and atomoxetine in general. In a study in which the effects of atomoxetine inhibitor on EEG and on children with ADHD were investigated, a few topographic changes in EEG bands and great increases at beta band after atomoxetine use were observed, and they concluded that they could return QEEG profile to normal to a large extent, and that atomoxetine caused a decrease in the absolute theta in the posterior region, and an increase in absolute beta (especially in the right and midline frontal regions), which was revealed in the EEG records that they received one hour after the application of atomoxetine, and that delta band showed an increase especially in the central regions, and beta band increased as a whole. They also concluded that there was not any significant stimulant effect on the alpha activity, atomoxetine had minimal long-term effects on QEEG changes in their present study, atomoxetine caused the normalization of QEEGs of those who responded to atomoxetine, but it had no effect on QEEGs of those who did not respond to atomoxetine $[1,8,30]$.

When the results of EEG analysis after atomoxetine use were evaluated, it was seen that EEG power spectrum densities in delta, theta, alpha and beta bands showed similarities to the findings obtained after methylphenidate use. Although the maximum power and frequencies at which electrodes were statistically significantly varied, they mostly occurred in the frontal and temporal regions for both methylphenidate and atomoxetine in general.

The clinical and neurophysiologic effects of the neural response to medical treatment of children with ADHD are still uncertain. Previous studies regarded a decrease in theta activity and an increase in beta activity as a positive neural response. Nevertheless, a strong correlation was reported between the increased beta activity in neural response and positive clinical results rather than the decrease of theta activity
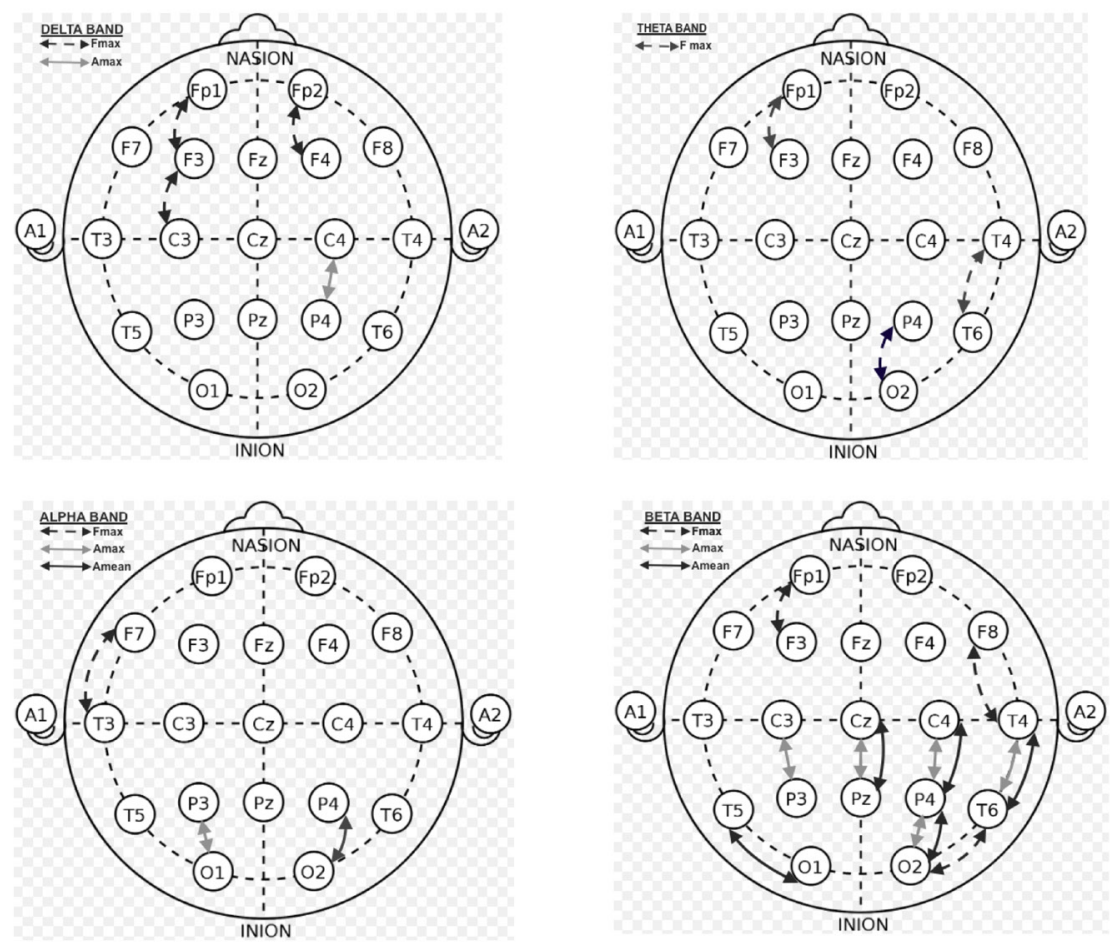

(a)
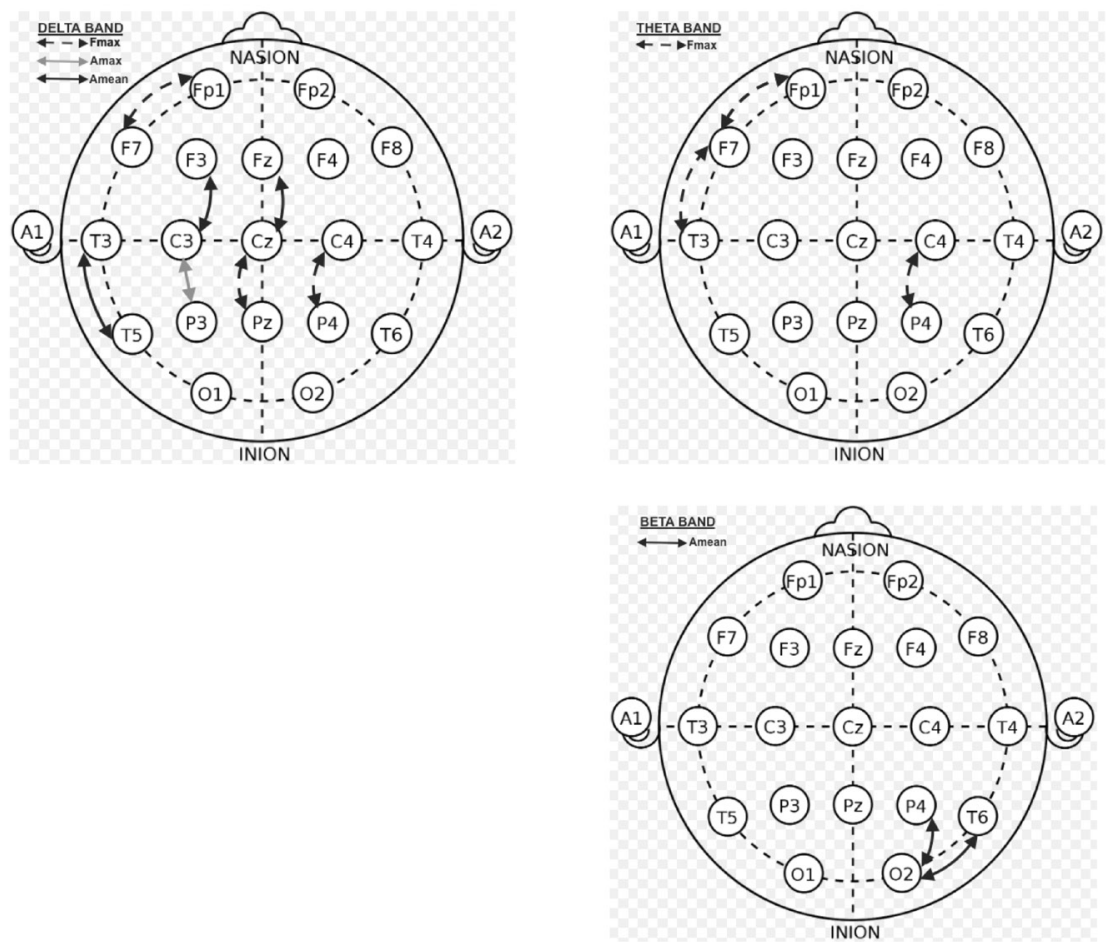

(b)

Figure 4. Representation of the statistically significant active electrodes according to the maximum frequen$c y$, maximum amplitude, and mean amplitude of power values (a) for the Methylphenidate using ADHD/Control groups, and (b) for the Atomoxetine using ADHD/Control groups. 
in children with $\mathrm{ADHD}$ [34]. The present study may also be consistent with findings in brain imaging studies. For example, decreased blood flow in the frontal regions, decreased metabolic activity, activation differences, and decreased frontal lobe volume were reported for many ADHD patients using neuroimaging studies [35-39].

In this study, QEEG was found statistically significant in the delta/beta ratio of $\mathrm{Fz}$ $\mathrm{Cz}$ electrode, and the delta/alpha ratio of C4-P4 electrode after treatment with both methylphenidate and atomoxetine. QEEG ratios, especially after atomoxetine treatment, were more statistically significant at the frontal electrodes than after methylphenidate treatment. These results suggest that both methylphenidate and atomoxetine are effective in treatment, and they have effect on the neural mechanisms in ADHD patients. The increase of spectral power values activity while the spectral power values of the delta activity decrease can be a sign suggesting that higher cortical functions such as attention, consciousness, and environmental awareness may be getting better. It is of importance to examine the relationship between the behavioral and cognitive functionality in patients and power spectra in EEG profiles. Finally, QEEG can be supported the $A D H D$ diagnosis along to drug treatment. At the same time, the clinician can also be used as an additive diagnostic tool.

\section{Disclosure Statement}

\section{Funding}

This work is supported by the scientific and technological research council of Turkey, [grant number: TUBITAK-115E832].

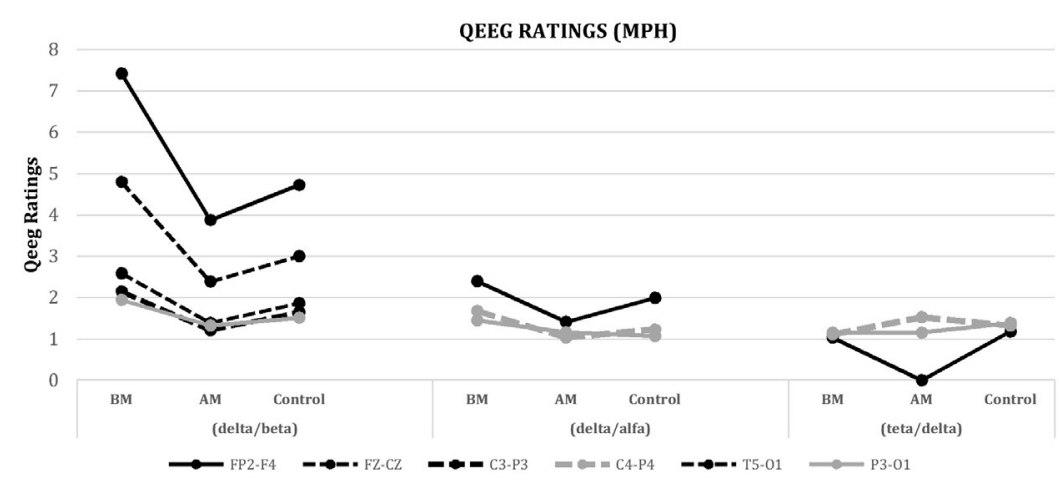

(BM:BeforeMethylphenidate AM: After Methylphenidate)

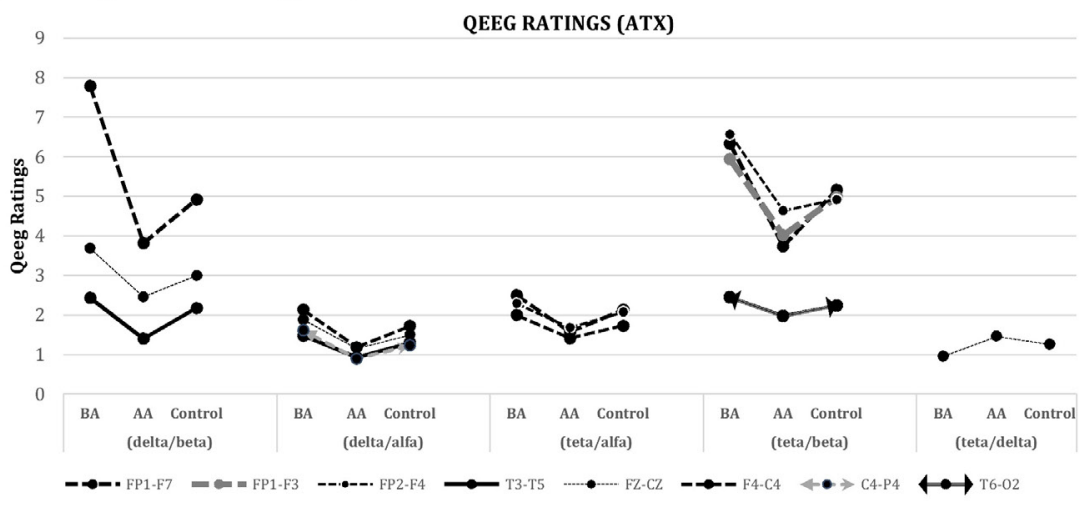

(b)

(BA : Beforeatomoxsetine A : After Atomoxsetive)

Figure 5. Statistical comparison of QEEG ratios after (a) Methylphenidate using ADHD and (b) Atomoxetine using ADHD

\section{Author Contributions}

R.A., E.D., M.T.: conception and design of the paper

E.D., S.O.: medical interpretation of the study, psychiatric evaluation of patients

A.K. B., M.C., H.P.: medical interpretation of the study, neurological evaluation of patients
R.A., M.T.: acquisition and analysis of data; drafting the manuscript or figures/tables 
Table 1. Statistical significance of EEG channels and QEEG ratios between ADHD group using Methylphenidate/ Atomoxetine and control group according to EEG frequency bands.

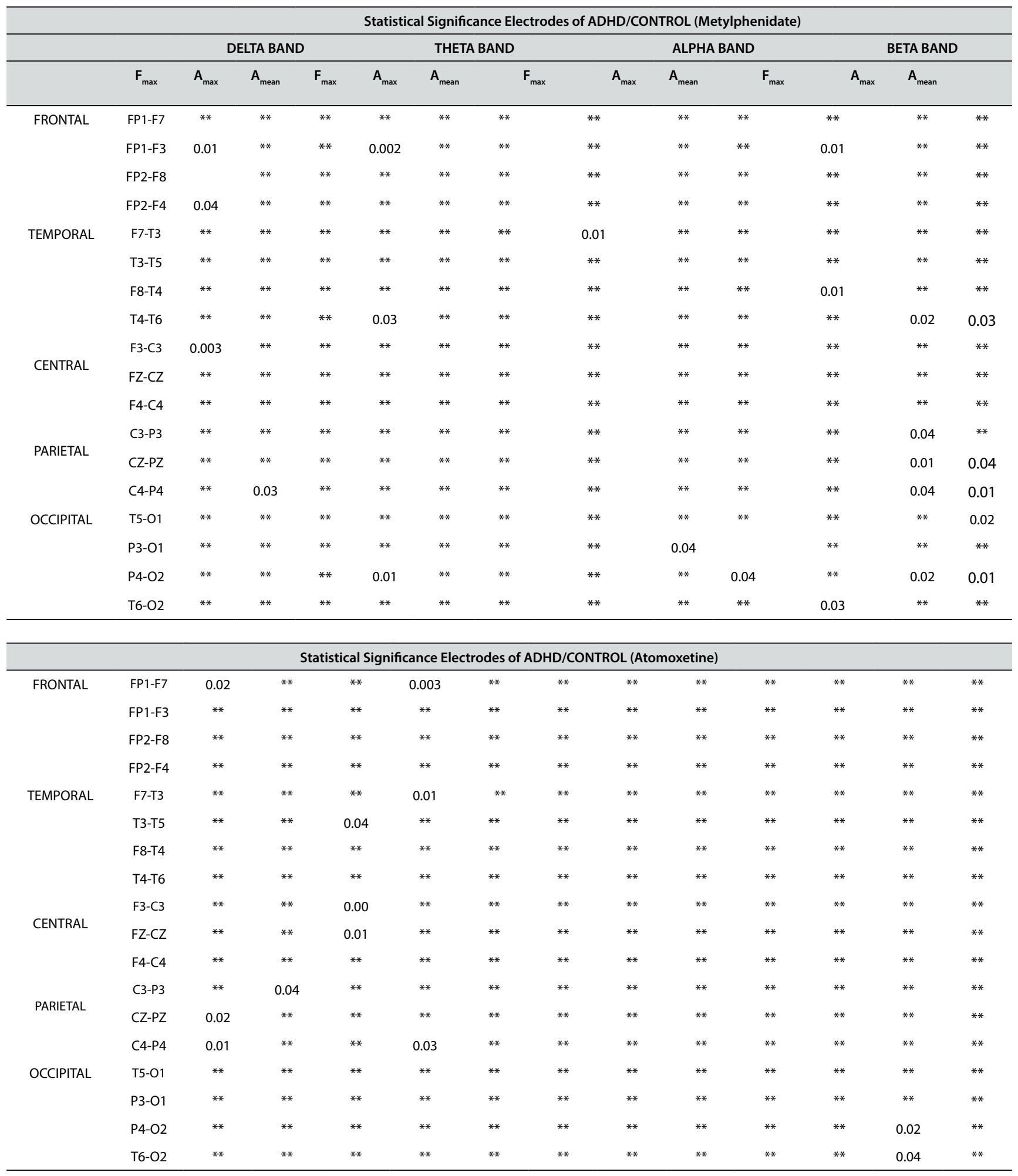




\begin{tabular}{|c|c|c|c|c|c|c|c|c|c|c|c|}
\hline & & \multicolumn{5}{|c|}{ QEEG RATINGS (Methylphenidate) } & \multicolumn{5}{|c|}{ QEEG RATINGS (Atomoxetine) } \\
\hline & & delta/beta & delta/alpha & theta/alpha & theta/beta & theta/delta & delta/beta & delta/alpha & theta/alpha & theta/beta & theta/delta \\
\hline \multirow[t]{4}{*}{ FRONTAL } & FP1-F7 & $* *$ & $* *$ & $* *$ & $* *$ & $* *$ & 0.03 & $* *$ & 0.01 & 0.03 & $* *$ \\
\hline & FP1-F3 & $* *$ & $* *$ & $* *$ & $* *$ & $* *$ & $* *$ & $* *$ & $* *$ & 0.02 & $* *$ \\
\hline & FP2-F8 & $* *$ & $* *$ & $* *$ & $* *$ & $* *$ & $* *$ & $* *$ & $* *$ & ** & ** \\
\hline & FP2-F4 & 0.02 & 0.02 & $* *$ & $* *$ & 0.02 & $* *$ & $* *$ & 0.03 & 0.02 & $* *$ \\
\hline \multirow[t]{5}{*}{ TEMPORAL } & F7-T3 & $* *$ & $* *$ & $* *$ & $* *$ & $* *$ & $* *$ & $* *$ & $* *$ & $* *$ & $* *$ \\
\hline & T3-T5 & $* *$ & $* *$ & $* *$ & $* *$ & $* *$ & 0.01 & 0.01 & $* *$ & $* *$ & $* *$ \\
\hline & F8-T4 & $* *$ & $* *$ & $* *$ & $* *$ & $* *$ & $* *$ & $* *$ & $* *$ & $* *$ & $* *$ \\
\hline & T4-T6 & $* *$ & $* *$ & $* *$ & $* *$ & $* *$ & $* *$ & $* *$ & $* *$ & $* *$ & $* *$ \\
\hline & F3-C3 & $* *$ & $* *$ & $* *$ & $* *$ & $* *$ & $* *$ & $* *$ & $* *$ & $* *$ & $* *$ \\
\hline \multirow{3}{*}{ CENTRAL } & $\mathrm{FZ}-\mathrm{CZ}$ & 0.04 & $* *$ & $* *$ & $* *$ & $* *$ & 0.02 & 0.03 & $* *$ & $* *$ & 0.04 \\
\hline & $\mathrm{F} 4-\mathrm{C} 4$ & $* *$ & $* *$ & $* *$ & $* *$ & $* *$ & $* *$ & 0.01 & 0.01 & $* *$ & $* *$ \\
\hline & C3-P3 & 0.03 & $* *$ & $* *$ & $* *$ & $* *$ & $* *$ & $* *$ & $* *$ & $* *$ & $* *$ \\
\hline \multirow[t]{2}{*}{ PARIETAL } & CZ-PZ & $* *$ & $* *$ & $* *$ & $* *$ & $* *$ & $* *$ & $* *$ & ** & $* *$ & $* *$ \\
\hline & C4-P4 & $* *$ & 0.03 & $* *$ & $* *$ & 0.02 & $* *$ & 0.001 & $* *$ & $* *$ & $* *$ \\
\hline \multirow[t]{4}{*}{ OCCIPITAL } & $\mathrm{T} 5-\mathrm{O} 1$ & 0.02 & $* *$ & $* *$ & $* *$ & $* *$ & $* *$ & $* *$ & $* *$ & $* *$ & $* *$ \\
\hline & P3-O1 & $* *$ & $* *$ & $* *$ & $* *$ & $* *$ & $* *$ & $* *$ & $* *$ & $* *$ & $* *$ \\
\hline & $\mathrm{P} 4-\mathrm{O} 2$ & 0.02 & 0.02 & $* *$ & $* *$ & 0.003 & $* *$ & $* *$ & $* *$ & $* *$ & $* *$ \\
\hline & $\mathrm{T} 6-\mathrm{O} 2$ & ** & ** & $* *$ & $* *$ & $* *$ & ** & $* *$ & $* *$ & 0.03 & ** \\
\hline
\end{tabular}

$\left(F_{\text {max }}:\right.$ The frequency at which the amplitude is maximum, $A_{\max }:$ Maximum power, $A_{\text {mean }}:$ Mean power, $\left.{ }^{* *}: p \geq 0.05\right)$

[1] Barry R.J.,Clarke A.R., Hajos M.,McCarthy R., SelikowitzM.,Bruggemann J.M., Acute atomoxetine effects on the EEG of children with attentiondeficit/hyperactivity disorder, Neuropharmacology, 2009, 57, 702707

[2] Barkley R., Attention-Deficit/Hyperactivity Disorder. The Guilford Press, A hand- book for diagnosis and treatment New York (770 pp).2006

[3] Ercan E.S., Akyol Ardic U., Kabukcu Basay B., Ercan E.,Basay O., Atomoxetine response in the inattentive and combined subtypes of attention deficit hyperactivity disorder: a retrospective chart review, Atten Defic Hyperact Disord, 2013, 5, 377-385

[4] Solanto M., Newcorn J., Vail L., Gilbert S., Ivanov I.,Lara R., Stimulant drug response in the predominantly inattentive and combined subtypes of attention-deficit/hyperactivity disorder, J Child Adolesc Psychopharmacol, 2009, 19, 663-671

[5] Sun L., Wang Y.F., He H.,Chen J., [Changes of the alpha competitive structure after administration of single dose methylphenidate in different subtypes of attention deficit hyperactivity disorder boys], Beijing Da Xue Xue Bao, 2007, 39, 289-292

[6] Clarke A.R., Barry R.J., Bond D., McCarthy R.,Selikowitz M., Effects of stimulant medications on the EEG of children with attention-deficit/ hyperactivity disorder, Psychopharmacology (Berl), 2002, 164, 277284
[7] Clarke A.R., Barry R.J., McCarthy R., Selikowitz M., Brown C.R.,Croft R.J., Effects of stimulant medications on the EEG of children with Attention-Deficit/Hyperactivity Disorder Predominantly Inattentive type, International Journal of Psychophysiology, 2003, 47, 129-137

[8] Clarke A.R., Barry R.J., McCarthy R., Selikowitz M.,Johnstone S.J., Effects of stimulant medications on the EEG of girls with AttentionDeficit/Hyperactivity Disorder, Clin Neurophysiol, 2007, 118, 27002708

[9] Lidzba K., Granstroem S., Leark R.A., Kraegeloh-Mann I.,Mautner V.F., Pharmacotherapy of attention deficit in neurofibromatosis type 1: effects on cognition, Neuropediatrics, 2014, 45, 240-246

[10] Chabot R.J., Merkin H., Wood L.M., Davenport T.L.,Serfontein G., Sensitivity and specificity of QEEG in children with attention deficit or specific developmental learning disorders, Clin Electroencephalogr, 1996, 27, 26-34

[11] Chabot R.J., Orgill A.A., Crawford G., Harris M.J.,Serfontein G., Behavioral and electrophysiologic predictors of treatment response to stimulants in children with attention disorders, J Child Neurol, 1999, 14, 343-351

[12] Chabot R.J., di Michele F., Prichep L.,John E.R., The clinical role of computerized EEG in the evaluation and treatment of learning and attention disorders in children and adolescents, J Neuropsychiatry Clin Neurosci, 2001, 13, 171-186 
[13] Clarke A.R., Barry R.J., McCarthy R.,Selikowitz M., Age and sex effects in the EEG: development of the normal child, Clin Neurophysiol, 2001, 112, 806-814

[14] Clarke A.R., Barry R.J., McCarthy R.,Selikowitz M., EEG-defined subtypes of children with attention-deficit/hyperactivity disorder, Clin Neurophysiol, 2001, 112, 2098-2105

[15] Clarke A.R., Barry R.J., McCarthy R.,Selikowitz M., Age and sex effects in the EEG: differences in two subtypes of attention-deficit/ hyperactivity disorder, Clin Neurophysiol, 2001, 112, 815-826

[16] Clarke A.R., Barry R.J., McCarthy R.,Selikowitz M., Electroencephalogram differences in two subtypes of attentiondeficit/hyperactivity disorder, Psychophysiology, 2001, 38, 212-221

[17] Clarke A.R., Barry R.J., McCarthy R.,Selikowitz M., Excess beta activity in children with attention-deficit/hyperactivity disorder: an atypical electrophysiological group, Psychiatry Res, 2001, 103, 205-218

[18] Clarke A.R., Barry R.J., McCarthy R.,Selikowitz M., Children with attention-deficit/hyperactivity disorder and comorbid oppositional defiant disorder: an EEG analysis, Psychiatry Res, 2002, 111, 181-190

[19] Clarke A.R., Barry R.J., McCarthy R., Selikowitz M., Clarke D.C.,Croft R.J., Effects of stimulant medications on children with attention-deficit/ hyperactivity disorder and excessive beta activity in their EEG, Clin Neurophysiol, 2003, 114, 1729-1737

[20] Clarke A.R., Barry R.J., McCarthy R., Selikowitz M., Clarke D.C.,Croft R.J., EEG activity in girls with attention-deficit/hyperactivity disorder, Clin Neurophysiol, 2003, 114, 319-328

[21] Clarke A.R., Barry R.J., McCarthy R., Selikowitz M., Magee C.A., Johnstone S.J., et al., Quantitative EEG in low-IQ children with attention-deficit/hyperactivity disorder, Clin Neurophysiol, 2006, 117, 1708-1714

[22] Loo S.K.,Makeig S., Clinical utility of EEG in attention-deficit/ hyperactivity disorder: a research update, Neurotherapeutics, 2012, 9, 569-587

[23] Song D.H., Shin D.W., Jon D.I.,Ha E.H., Effects of methylphenidate on quantitative EEG of boys with attention-deficit hyperactivity disorder in continuous performance test, Yonsei Med J, 2005, 46, 34-41

[24] Woestenburg J.C., Verbaten M.N.,Slangen J.L., The removal of the eye-movement artifact from the EEG by regression analysis in the frequency domain, Biol Psychol, 1983, 16, 127-147

[25] Kenemans J.L., Molenaar P.C., Verbaten M.N.,Slangen J.L., Removal of the ocular artifact from the EEG: a comparison of time and frequency domain methods with simulated and real data, Psychophysiology, 1991, 28, 114-121

[26] Croft R.J.,Barry R.J., Removal of ocular artifact from the EEG: a review, Neurophysiol Clin, 2000, 30, 5-19

[27] Fatourechi M., Bashashati A., Ward R.K.,Birch G.E., EMG and EOG artifacts in brain computer interface systems: A survey, Clin Neurophysiol, 2007, 118, 480-494

[28] Swartwood M.O., Swartwood J.N., Lubar J.F., Timmermann D.L., Zimmerman A.W.,Muenchen R.A., Methylphenidate effects on EEG, behavior, and performance in boys with ADHD, Pediatr Neurol, 1998, $18,244-250$

[29] Lubar J.F., White J.N., Jr., Swartwood M.O.,Swartwood J.N., Methylphenidate effects on global and complex measures of EEG, Pediatr Neurol, 1999, 21, 633-637

[30] Chiarenza G.A., Chabot R., Isenhart R., Montaldi L., Chiarenza M.P., Torto M.G., et al., The quantified EEG characteristics of responders and non-responders to long-term treatment with atomoxetine in children with attention deficit hyperactivity disorders, Int J Psychophysiol, 2016, 104, 44-52

[31] Ahmadlou M.,Adeli H., Wavelet-synchronization methodology: a new approach for EEG-based diagnosis of ADHD, Clin EEG Neurosci, 2010, 41, 1-10

[32] Vles J.S., Feron F.J., Hendriksen J.G., Jolles J., van Kroonenburgh M.J.,Weber W.E., Methylphenidate down-regulates the dopamine receptor and transporter system in children with attention deficit hyperkinetic disorder (ADHD), Neuropediatrics, 2003, 34, 77-80

[33] Loo S.K., Teale P.D.,Reite M.L., EEG correlates of methylphenidate response among children with ADHD: a preliminary report, Biological psychiatry, 1999, 45, 1657-1660

[34] Janssen T.W.P., Bink M., Weeda W.D., Gelade K., van Mourik R., Maras A., et al., Learning curves of theta/beta neurofeedback in children with ADHD, Eur Child Adolesc Psychiatry, 2017, 26, 573-582

[35] Zametkin A.J., Nordahl T.E., Gross M., King A.C., Semple W.E., Rumsey J., et al., Cerebral glucose metabolism in adults with hyperactivity of childhood onset, N Engl J Med, 1990, 323, 1361-1366

[36] Sowell E.R., Thompson P.M., Welcome S.E., Henkenius A.L., Toga A.W.,Peterson B.S., Cortical abnormalities in children and adolescents with attention-deficit hyperactivity disorder, Lancet, 2003, 362, 16991707

[37] Bush G., Valera E.M.,Seidman L.J., Functional neuroimaging of attention-deficit/hyperactivity disorder: a review and suggested future directions, Biol Psychiatry, 2005, 57, 1273-1284

[38] Dickstein S.G., Bannon K., Castellanos F.X.,Milham M.P., The neural correlates of attention deficit hyperactivity disorder: an ALE metaanalysis, J Child Psychol Psychiatry, 2006, 47, 1051-1062

[39] Cortese S., The neurobiology and genetics of Attention-Deficit/ Hyperactivity Disorder (ADHD): what every clinician should know, Eur J Paediatr Neurol, 2012, 16, 422-433 